\title{
Advanced Mirror Technology Development (AMTD) Thermal Trade Studies
}

\author{
Thomas Brooks*a \\ aNASA Marshall Space Flight Center, AL, USA 35812
}

\begin{abstract}
Advanced Mirror Technology Development (AMTD) is being done at Marshall Space Flight Center (MSFC) in preparation for the next large aperture UVOIR space observatory. A key science mission of that observatory is the detection and characterization of 'Earth-like' exoplanets. Direct exoplanet observation requires a telescope to see a planet which will be $10^{-10}$ times dimmer than its host star. To accomplish this using an internal coronagraph requires a telescope with an ultra-stable wavefront error (WFE). This paper investigates parametric relationships between primary mirror physical parameters and thermal WFE stability.

Candidate mirrors are designed as a mesh and placed into a thermal analysis model to determine the temperature distribution in the mirror when it is placed inside of an actively controlled cylindrical shroud at Lagrange point 2. Thermal strains resulting from the temperature distribution are found and an estimation of WFE is found to characterize the effect that thermal inputs have on the optical quality of the mirror. This process is repeated for several mirror material properties, material types, and mirror designs to determine how to design a mirror for thermal stability.
\end{abstract}

